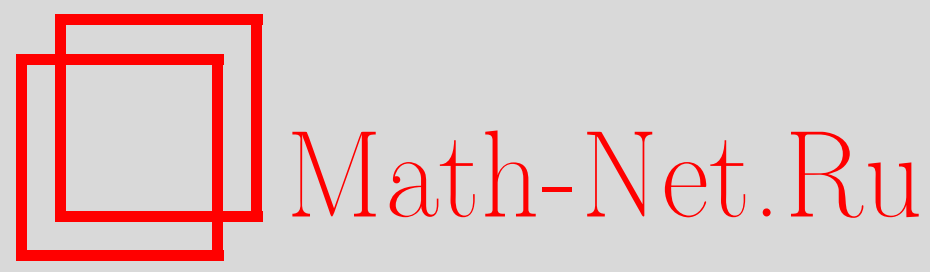

M. М. Фаддеев, Р. Г. Штеренберг, О подобии некоторых дифференциальных операторов самосопряженным, Матем. заметки, 2002, том 72, выпуск 2, 292-302

DOI: https://doi.org/10.4213/mzm423

Использование Общероссийского математического портала Math-Net.Ru подразумевает, что вы прочитали и согласны с пользовательским соглашением http://www.mathnet.ru/rus/agreement

Параметры загрузки:

IP : 54.92 .164 .108

26 апреля 2023 г., $14: 21: 43$

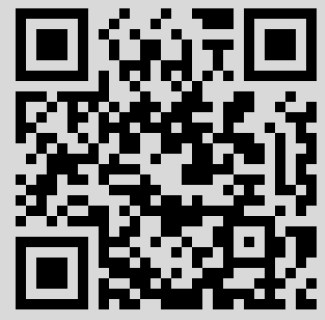




\title{
О ПОДОБИИ НЕКОТОРЫХ ДИФФЕРЕНЦИАЛЬНЫХ ОПЕРАТОРОВ САМОСОПРЯЖЕННЫМ
}

\author{
М. М. Фаддеев, Р. Г. Штеренберг
}

Работа посвящена исследованию задачи о подобии самосопряженному оператору операторов вида $L=-\frac{\operatorname{sign} x}{|x|^{\alpha} p(x)} \frac{d^{2}}{d x^{2}}, \alpha>-1$, в пространстве $L_{2}(\mathbb{R})$ с весом $|x|^{\alpha} p(x)$. Известен положительный ответ в данной задаче в случае $p(x) \equiv 1$, полученный на основе тонких методов теории гильбертовых пространств с индефинитной метрикой. Использование общего критерия подобия в сочетании с методами теории возмущений дифференциальных операторов позволяет обобщить данный результат на значительно более широкий класс весовых функций $p(x)$.

Библиография: 13 названий.

1. Введение. Говорят, что оператор L подобен самосопряженному, если существуют самосопряженньй оператор $A$ и ограниченный, ограниченно обратимый оператор $X$ такие, что $L=X^{-1} A X$. В настоящей работе мы рассматриваем вопрос о подобии некоторых дифференциальных операторов самосопряженным. Именно, будут получены результаты о подобии самосопряженным операторов вида

$$
L=-\frac{\operatorname{sign} x}{|x|^{\alpha} p(x)} \frac{d^{2}}{d x^{2}}, \quad \alpha>-1, \quad 0<c<p(x)<C<+\infty,
$$

рассматриваемых в пространстве функций, квадратично суммируемых на оси с весом $p(x)|x|^{\alpha}$, на естественной области определения $\mathscr{D}(L)$, состоящей из тех функций $f \in$ $L_{2}\left(p(x)|x|^{\alpha}, \mathbb{R}\right)$, которые абсолютно непрерьвны вместе со своими первыми производными, причем $L f \in L_{2}\left(p(x)|x|^{\alpha}, \mathbb{R}\right)$. Случаю $p(x) \equiv 1$ посвящена работа [1], в которой доказательство подобия было проведено с использованием методов теории гильбертовых пространств с индефинитной метрикой (см., например, [2]). Случаю $\alpha=0$ посвящена работа авторов [3], в которой рассматриваются возмущения операторов рассматриваемого вида быстро убывающим потенциалом. В работе [4] рассматривается задача о подобии самосопряженному для операторов вида $-(\operatorname{sign} x) p\left(d^{2} / d x^{2}\right)$, где $p$ - полином.

Интерес к операторам указанного вида определяется тем, что известные достаточные (а для диссипативных операторов - и необходимые) условия подобия самосопряженньм, выражаемые в терминах характеристической функции оператора (см. [5]-[7]), для данных операторов не вьполняются, несмотря на то, что данные операторы имеют только абсолютно непрерывный спектр. Затруднительно в данной задаче использовать

Работа выполнена при поддержке Российского фонда фундаментальных исследований, гранты № 96-15-96103 и № 00-01-00479. 
и технику доказательства ограниченности спектральных проекторов оператора, удобную для рассмотрения вопроса о подобии самосопряженному оператору дифференциальных операторов с быстро убьвающим невещественным потенциалом.

Наши рассмотрения будут опираться на обший интегрально-резольвентньй критерий подобия (см. [8], [9]), который заключается в том, что оператор $L$ в гильбертовом пространстве $H$ подобен самосопряљенному оператору тогда и только тогда, когда его спектр вещественен и выполнены оценки

$$
\begin{gathered}
\sup _{\varepsilon>0} \varepsilon \int_{-\infty}^{+\infty}\left\|(L-z)^{-1} u\right\|^{2} d \xi \leqslant C\|u\|^{2}, \\
\sup _{\varepsilon>0} \varepsilon \int_{-\infty}^{+\infty}\left\|\left(L^{*}-z\right)^{-1} u\right\|^{2} d \xi \leqslant C\|u\|^{2}, \quad z=\xi+i \varepsilon, \quad u \in H .
\end{gathered}
$$

Удобство использования этого критерия обусловлено тем, что исследуемые операторы представляют собой вполне несамосопряженные расширения симметричных операторов с совпадающими индексами дефекта равньми 2. Тем самым, как и при исследовании вопроса подобия самосопряженному оператору конечномерных возмущений оператора умножения на независимую переменную (операторов модели Фридрихса, см., например, [9]), оказывается возможным провести требуемую оценку резольвенты иследуемого оператора.

2. Случай “чисто степенного” веса. Здесь мы, используя критерий (1), докажем следующую теорему.

Теорема 1 (Фляйге, Найман [1]). Onератор

$$
L_{0}=-\frac{\operatorname{sign} x}{|x|^{\alpha}} \frac{d^{2}}{d x^{2}}, \quad \alpha>-1
$$

рассматриваемый в пространстве $L_{2}\left(|x|^{\alpha}, \mathbb{R}\right)$ на естественной области определения $\mathscr{D}\left(L_{0}\right)$, состоящей из локально абсолютно непрерывных вместе со своими первыми производными функиий $f$, для которых $f, L_{0} f \in L_{2}\left(|x|^{\alpha}, \mathbb{R}\right)$, подобен самосопря⿻енному.

ДокАЗАТЕЛЬСТво. Суть доказательства состоит в том, что действие резольвенты исследуемого оператора $L_{0}$ мы сведем к действию резольвент некоторых вспомогательных самосопряженных операторов, для которых оценки (2), очевидно, вьполнены.

Итак, введем в рассмотрение следующие самосопряженные операторы:

$$
L_{1}=\frac{1}{|x|^{\alpha}} \frac{d^{2}}{d x^{2}}, \quad L_{2}=-\frac{1}{|x|^{\alpha}} \frac{d^{2}}{d x^{2}}, \quad L_{3}=-\frac{1}{|x|^{\alpha}} \frac{d}{d x} \operatorname{sign} x \frac{d}{d x}
$$

с естественными областями определения. Для того чтобы выписать действие их резольвент и резольвенты оператора $L_{0}$, рассмотрим функции $\varphi_{1}, \psi_{1}, \varphi_{2}, \psi_{2}$ такие, что

$$
\begin{gathered}
L_{1} \varphi_{1}(x, \widetilde{\varrho})=\lambda \varphi_{1}(x, \widetilde{\varrho}), \quad L_{1} \psi_{1}(x, \widetilde{\varrho})=\lambda \psi_{1}(x, \widetilde{\varrho}), \\
\varphi_{1}(x) \stackrel{x \rightarrow-\infty}{\longrightarrow} 0, \quad \psi_{1}(x) \stackrel{x \rightarrow+\infty}{\longrightarrow} 0, \quad \widetilde{\varrho}^{2}=\lambda, \quad \operatorname{Re} \widetilde{\varrho}>0, \\
L_{2} \varphi_{2}(x, \varrho)=\lambda \varphi_{2}(x, \varrho), \quad L_{2} \psi_{2}(x, \varrho)=\lambda \psi_{2}(x, \varrho), \\
\varphi_{2}(x) \stackrel{x \rightarrow-\infty}{\longrightarrow} 0, \quad \psi_{2}(x) \stackrel{x \rightarrow+\infty}{\longrightarrow} 0, \quad \varrho^{2}=\lambda, \quad \operatorname{Im} \varrho>0 .
\end{gathered}
$$


Поскольку весовая функция $|x|^{\alpha}$ является четной, то выполнены равенства

$$
\varphi_{1}(x)=\psi_{1}(-x), \quad \varphi_{2}(x)=\psi_{2}(-x)
$$

Кроме того, известно, что

$$
\begin{array}{ll}
\psi_{1}(x, \widetilde{\varrho}) \sim \frac{1}{\left(\left(\widetilde{\varrho}^{2 /(2+\alpha)} x\right)^{|\alpha| / 4}+1\right)^{\operatorname{sign} \alpha}} \exp \left\{-\widetilde{\varrho} \frac{1}{\alpha / 2+1} x^{\alpha / 2+1}\right\}, \quad x \rightarrow+\infty, \\
\psi_{2}(x, \varrho) \sim \frac{1}{\left(\left(\varrho^{2 /(2+\alpha)} x\right)^{|\alpha| / 4}+1\right)^{\operatorname{sign} \alpha}} \exp \left\{i \varrho \frac{1}{\alpha / 2+1} x^{\alpha / 2+1}\right\}, \quad x \rightarrow+\infty .
\end{array}
$$

Также легко показать, что два линейно независимых решения дифференциального уравнения $L_{2} y(x)=y(x)$ представимы абсолютно сходящимися рядами

$$
y_{1}(x)=\sum_{k=0}^{\infty} C_{k} x^{k(2+\alpha)}, \quad y_{2}(x)=\sum_{k=0}^{\infty} D_{k} x^{k(2+\alpha)+1}, \quad x \geqslant 0
$$

где

$$
\begin{gathered}
C_{0} \neq 0, \quad D_{0} \neq 0, \\
C_{k}=\frac{(-1)^{k} C_{0}}{k !(2+\alpha)^{2 k}} \frac{\Gamma(1-1 /(2+\alpha))}{\Gamma(k+1-1 /(2+\alpha))}, \quad k \geqslant 1 . \\
D_{k}=\frac{(-1)^{k} D_{0}}{k !(2+\alpha)^{2 k}} \frac{\Gamma(1+1 /(2+\alpha))}{\Gamma(k+1+1 /(2+\alpha))},
\end{gathered}
$$

Рассмотрим, кроме того, функции $\varphi(x)$ и $\varphi_{3}(x)$, удовлетворяющие уравнениям $L_{0} \varphi(x)$ $=\lambda \varphi(x), L_{3} \varphi_{3}(x)=\lambda \varphi_{3}(x)$ и совпадаюшие на отрицательной полуоси $\mathbb{R}_{-}$с функцией $\varphi_{1}(x)$. Так как оператор $L_{2}$, будучи самосопряженным, не имеет невешественных собственных значений, то функции $\varphi_{2}(x)$ и $\psi_{2}(x)$ линейно независимы. Поэтому на положительной полуоси $\mathbb{R}_{+}$

$$
\varphi(x)=A \varphi_{2}(x)+B \psi_{2}(x) \quad \text { и } \quad \varphi_{3}(x)=A_{3} \varphi_{2}(x)+B_{3} \psi_{2}(x), \quad \operatorname{Im} \lambda \neq 0,
$$

где константы $A, B, A_{3}, B_{3}$ определяются из условий сшивки согласно включениям $\varphi \in$ $\mathscr{D}\left(L_{0}\right), \varphi_{3} \in \mathscr{D}\left(L_{3}\right)$ :

$$
\begin{array}{rlrl}
A & =\frac{W}{W_{2}}, \quad B & =\frac{1}{W_{2}}\left(\varphi_{2}(0) \varphi_{1}^{\prime}(0)-\varphi_{2}^{\prime}(0) \varphi_{1}(0)\right), \\
A_{3} & =\frac{W_{3}}{W_{2}}, \quad B_{3}=-\frac{1}{W_{2}}\left(\varphi_{1}(0) \varphi_{2}^{\prime}(0)+\varphi_{1}^{\prime}(0) \varphi_{2}(0)\right)
\end{array}
$$

здесь мы используем обозначения

$$
\begin{aligned}
W & =\varphi_{1}(0) \psi_{2}^{\prime}(0)-\varphi_{1}^{\prime}(0) \psi_{2}(0)=\varphi(x) \psi^{\prime}(x)-\varphi^{\prime}(x) \psi(x) \\
W_{3} & =\varphi_{1}(0) \psi_{2}^{\prime}(0)+\varphi_{1}^{\prime}(0) \psi_{2}(0)=\operatorname{sign} x\left(\varphi_{3}(x) \psi_{3}^{\prime}(x)-\varphi_{3}^{\prime}(x) \psi_{3}(x)\right) \\
W_{2} & =\varphi_{2}(0) \psi_{2}^{\prime}(0)-\varphi_{2}^{\prime}(0) \psi_{2}(0)=\varphi_{2}(x) \psi_{2}^{\prime}(x)-\varphi_{2}^{\prime}(x) \psi_{2}(x)
\end{aligned}
$$


Аналогично, функции $\psi(x), \psi_{3}(x)$, удовлетворяющие уравнениям $L_{0} \psi(x)=\lambda \psi(x)$, $L_{3} \psi_{3}(x)=\lambda \psi_{3}(x)$ и совпадающие на $\mathbb{R}_{+}$с функцией $\psi_{2}(x)$, определяются на $\mathbb{R}_{-}$формулами

$$
\begin{gathered}
\psi(x)=C \varphi_{1}(x)+D \psi_{1}(x) \text { и } \psi_{3}(x)=C_{3} \varphi_{1}(x)+D_{3} \psi_{1}(x), \quad \operatorname{Im} \lambda \neq 0, \\
C=\frac{1}{W_{1}}\left(\psi_{2}(0) \psi_{1}^{\prime}(0)-\psi_{2}^{\prime}(0) \psi_{1}(0)\right), \quad D=\frac{W}{W_{1}} \\
C_{3}=\frac{1}{W_{1}}\left(\psi_{1}(0) \psi_{2}^{\prime}(0)+\psi_{1}^{\prime}(0) \psi_{2}(0)\right), \quad D_{3}=-\frac{W_{3}}{W_{1}} \\
W_{1}=\varphi_{1}(0) \psi_{1}^{\prime}(0)-\varphi_{1}^{\prime}(0) \psi_{1}(0)=\varphi_{1}(x) \psi_{1}^{\prime}(x)-\varphi_{1}^{\prime}(x) \psi_{1}(x) .
\end{gathered}
$$

Как легко теперь видеть, резольвенты рассматриваемых операторов могут быть записаны следуюшим образом:

$$
\begin{aligned}
& {\left[\left(L_{1}-\lambda\right)^{-1} u\right](x)=\frac{1}{W_{1}}\left(\psi_{1}(x) \int_{-\infty}^{x}|y|^{\alpha} \varphi_{1}(y) u(y) d y\right.} \\
& \left.\quad+\varphi_{1}(x) \int_{x}^{\infty}|y|^{\alpha} \psi_{1}(y) u(y) d y\right), \quad \operatorname{Im} \lambda \neq 0, \\
& {\left[\left(L_{2}-\lambda\right)^{-1} u\right](x)=-\frac{1}{W_{2}}\left(\psi_{2}(x) \int_{-\infty}^{x}|y|^{\alpha} \varphi_{2}(y) u(y) d y\right.} \\
& \left.\quad+\varphi_{2}(x) \int_{x}^{\infty}|y|^{\alpha} \psi_{2}(y) u(y) d y\right), \quad \operatorname{Im} \lambda \neq 0, \\
& {\left[\left(L_{3}-\lambda\right)^{-1} u\right](x)=-\frac{\operatorname{sign} x}{W_{3}}\left(\psi_{3}(x) \int_{-\infty}^{x} \operatorname{sign} y \cdot|y|^{\alpha} \varphi_{3}(y) u(y) d y\right.} \\
& \left.\quad+\varphi_{3}(x) \int_{x}^{\infty} \operatorname{sign} y \cdot|y|^{\alpha} \psi_{3}(y) u(y) d y\right), \quad \lambda:\left\{\operatorname{Im} \lambda \neq 0, W_{3}(\varrho, \widetilde{\varrho}) \neq 0\right\} \\
& {\left[\left(L_{0}-\lambda\right)^{-1} u\right](x)=-\frac{1}{W}\left(\psi(x) \int_{-\infty}^{x} \operatorname{sign} y \cdot|y|^{\alpha} \varphi(y) u(y) d y\right.} \\
& \left.\quad+\varphi(x) \int_{x}^{\infty} \operatorname{sign} y \cdot|y|^{\alpha} \psi(y) u(y) d y\right), \quad \lambda:\{\operatorname{Im} \lambda \neq 0, W(\varrho, \widetilde{\varrho}) \neq 0\} .
\end{aligned}
$$

Воспользуемся интегрально-резольвентным критерием подобия (1). Так как операторы $L_{1}, L_{2}, L_{3}$ самосопряженные, то для них оценки (1) справедливы. Рассматривая действие резольвент (17)-(20) на функциях с носителями, сосредоточенными на полуосях $\mathbb{R}_{ \pm}$, и принимая во внимание только что сделанное замечание, нетрудно установить, что для подобия оператора $L_{0}$ самосопряженному достаточно проверить выполнение оценок

$$
\left|\frac{W_{3}}{W}\right| \leqslant C^{\prime}, \quad\left|\frac{B W_{3}}{B_{3} W}\right| \leqslant C^{\prime}, \quad\left|\frac{C W_{3}}{C_{3} W}\right| \leqslant C^{\prime}, \quad \operatorname{Im} \varrho \geqslant 0, \quad \operatorname{Re} \varrho \geqslant 0, \quad \widetilde{\varrho}=\varrho,
$$

с некоторой константой $C^{\prime}$.

В самом деле, оценки (21) обеспечивают для оператора $L_{0}$ справедливость первого из неравенств (1); для доказательства второго неравенства достаточно заметить, что резольвента сопряженного оператора определяется формулой (20) (см. также (9)-(16)), где константы $A, B, C, D$ берутся с противоположным знаком. 
Равенства (4), (10)-(12) и (15) показывают, что условие (21) эквивалентно неравенству

$$
\frac{\left|\psi_{1}(0) \psi_{2}^{\prime}(0)-\psi_{1}^{\prime}(0) \psi_{2}(0)\right|}{\left|\psi_{1}(0) \psi_{2}^{\prime}(0)+\psi_{1}^{\prime}(0) \psi_{2}(0)\right|} \leqslant \text { Const, } \operatorname{Im} \varrho \geqslant 0, \quad \operatorname{Re} \varrho \geqslant 0, \quad \widetilde{\varrho}=\varrho .
$$

Теперь утверждение теоремы 1 вытекает из следующей леммы.

ЛЕмма 2. В условиях теоремы 1 справедливы равенства

$\frac{\psi_{1}(0) \psi_{2}^{\prime}(0)-\psi_{1}^{\prime}(0) \psi_{2}(0)}{\psi_{1}(0) \psi_{2}^{\prime}(0)+\psi_{1}^{\prime}(0) \psi_{2}(0)}=\frac{1-\exp \{i \pi /(2+\alpha)\}}{1+\exp \{i \pi /(2+\alpha)\}}, \quad \operatorname{Im} \varrho \geqslant 0, \quad \operatorname{Re} \varrho \geqslant 0, \quad \widetilde{\varrho}=\varrho$,

$\frac{\psi_{1}(0) \psi_{2}^{\prime}(0)-\psi_{1}^{\prime}(0) \psi_{2}(0)}{\psi_{1}(0) \psi_{2}^{\prime}(0)+\psi_{1}^{\prime}(0) \psi_{2}(0)}=\frac{1-\exp \{-i \pi /(2+\alpha)\}}{1+\exp \{-i \pi /(2+\alpha)\}}, \quad \operatorname{Im} \varrho \geqslant 0, \quad \operatorname{Re} \varrho \leqslant 0, \quad \widetilde{\varrho}=-\varrho$.

ДокАЗАТЕЛЬСТво. Функция $\psi_{2}(x)$ удовлетворяет при $x \geqslant 0$ дифференциальному уравнению $\psi_{2}^{\prime \prime}(x)=-\lambda x^{\alpha} \psi_{2}(x)$, и, следовательно, мы можем рассматривать $\psi_{2}(z, \varrho)$ как аналитическое решение уравнения $\psi_{2}^{\prime \prime}(z)=-\lambda z^{\alpha} \psi_{2}(z)$ в комплексной плоскости $\mathbb{C}$ с разрезом, проходящим по отрицательной полуоси $\mathbb{R}_{-}$. Аналогично, функция $\psi_{1}(z, \widetilde{\varrho})$ удовлетворяет уравнению $\psi_{1}^{\prime \prime}(z)=\lambda z^{\alpha} \psi_{1}(z)$, или после замены переменной $z \mapsto$ $\exp \{-i \pi /(2+\alpha)\} z$

$$
\psi_{1}^{\prime \prime}\left(\exp \left\{-i \frac{\pi}{2+\alpha}\right\} z\right)=-\lambda z^{\alpha} \psi_{1}\left(\exp \left\{-i \frac{\pi}{2+\alpha}\right\} z\right) .
$$

Известно, что формула (6) определяет асимптотику решения $\psi_{2}(z)$ при $|z| \rightarrow \infty$ во всем секторе убывания, содержащем $\mathbb{R}_{+}$. Легко видеть, что в области $\{\operatorname{Im} \varrho>0, \operatorname{Re} \varrho>0$, $\widetilde{\varrho}=\varrho\}$ луч $z=\exp \{i \pi /(2+\alpha)\} r, r \geqslant 0$, попадает в данньй сектор. Таким образом, функция $\psi_{2}(z)$ одновременно с $\psi_{1}(\exp \{-i \pi /(2+\alpha)\} z)$ убывает в направлении рассматриваемого луча. Поскольку линейно независимое с $\psi_{2}(z)$ решение имеет здесь растущую асимптотику

$$
y(z) \sim \frac{1}{\left(\left(\varrho^{2 /(2+\alpha)} z\right)^{|\alpha| / 4}+1\right)^{\operatorname{sign} \alpha}} \exp \left\{-i \varrho \frac{1}{\alpha / 2+1} z^{\alpha / 2+1}\right\}, \quad x \rightarrow+\infty,
$$

то из предыдущих рассуждений вытекает равенство

$$
\psi_{2}(z)=\psi_{1}\left(\exp \left\{-i \frac{\pi}{2+\alpha}\right\} z\right)
$$

Отметим, что $\psi_{1}(0) \neq 0$ и $\psi_{1}^{\prime}(0) \neq 0$, если $\operatorname{Re} \widetilde{\varrho}>0$. В самом деле, если верно обратное, то один из отрицательных операторов, заданных в пространстве $L_{2}\left(x^{\alpha}, \mathbb{R}_{+}\right)$ дифференциальным выражением

$$
D=\frac{1}{x^{\alpha}} \frac{d^{2}}{d x^{2}}
$$

на области определения, состоящей из абсолютно непрерьвных вместе со своими первыми производными функций $f(x)$ таких, что $f, D f \in L_{2}\left(x^{\alpha}, \mathbb{R}_{+}\right)$, и удовлетворяющих либо условию типа Дирихле $f(0)=0$, либо условию типа Неймана $f^{\prime}(0)=0$, имеет собственное значение $\lambda=\widetilde{\varrho}^{2}$, и мы приходим к противоречию.

Теперь из равенства (25) и только что доказанного утверждения легко следует тождество (22). Чтобы получить (23), необходимо в приведенном выше построении вместо замены переменных $z \mapsto \exp \{-i \pi /(2+\alpha)\} z$ осуществить замену $z \mapsto \exp \{+i \pi /(2+\alpha)\} z$.

Таким образом, лемма 2, а с ней и теорема 1 полностью доказаны. 
3. Общий случай. Перейдем теперь к рассмотрению оператора

$$
L=-\frac{\operatorname{sign} x}{|x|^{\alpha} p(x)} \frac{d^{2}}{d x^{2}}
$$

на уже описанной во введении естественной области определения $\mathscr{D}(L)$ в пространстве $L_{2}\left(p(x)|x|^{\alpha}, \mathbb{R}\right)$. Основной результат настоящей работы заключается в справедливости следующей теоремы.

Теорема 3. Пусть $\alpha>-1$. Предположим, что весовая функиия $p(x)$ абсолютно непрерывна вместе со своей первой производной и удовлетворяет двусторонней оценке $0<c \leqslant p(x) \leqslant C<+\infty$. Предположим также, что выполнены следующие условия:

$$
\begin{gathered}
\int_{-\infty}^{+\infty} \frac{|x|^{\alpha}|p(x)-1|}{\left(|x|^{|\alpha| / 2}+1\right)^{\operatorname{sign} \alpha}} d x<\infty \\
\int_{-\infty}^{+\infty} \frac{\left|3\left(u^{\prime \prime}(x)\right)^{2} / 2 u^{\prime}(x)-u^{\prime \prime \prime}(x)\right|}{\left(u^{\prime}(x)\right)^{2}\left(|u(x)|^{|\alpha| / 2}+1\right)^{\operatorname{sign} \alpha}} d x<\infty
\end{gathered}
$$

$2 \partial e$

$$
u(x)=\operatorname{sign} x\left(\left.\left|\left(\frac{\alpha}{2}+1\right) \int_{0}^{x} \sqrt{p(y)}\right| y\right|^{\alpha / 2} d y \mid\right)^{1 /(\alpha / 2+1)} .
$$

Тогда оператор $L$ подобен самосопрязенному оператору.

Выполнение условий (26) и (27), как будет видно из доказательства, позволит нам для анализа оператора $L$, с учетом полученного результата для оператора $L_{0}$, реализовать схему исследования, аналогичную применяемой при рассмотрении операторов Шрёдингера с убывающим потенциалом [10]-[13]. Сами же эти условия вьполняются в достаточно широком классе весовых функций $p(x)$, к примеру, для любой гладкой функции, равной 1 тождественно в некоторой окрестности бесконечности или достаточно быстро стремящейся к 1 на $\pm \infty$.

ДокАЗАТЕльство. Доказательству теоремы предпошлем несколько лемм.

Лемма 4. Oператор L не имеет невещественных собственных значений.

ДокАЗАтЕльство. Так же, как и по ходу доказательства теоремы 1 , мы введем в рассмотрение вспомогательные самосопряженные операторы $L_{1}^{p}, L_{2}^{p}, L_{3}^{p}$ и решения соответствующих дифференциальных уравнений. Итак, рассмотрим операторы

$$
L_{1}^{p}=\frac{1}{|x|^{\alpha} p(x)} \frac{d^{2}}{d x^{2}}, \quad L_{2}^{p}=-\frac{1}{|x|^{\alpha} p(x)} \frac{d^{2}}{d x^{2}}, \quad L_{3}^{p}=-\frac{1}{|x|^{\alpha} p(x)} \frac{d}{d x} \operatorname{sign} x \frac{d}{d x}
$$

с естественными областями определения в пространстве $L_{2}\left(|x|^{\alpha} p(x), \mathbb{R}\right)$ и функции $\psi_{1,2}^{p}$, $\varphi_{1,2}^{p}$, удовлетворяющие условиям

$$
\begin{gathered}
L_{1}^{p} \varphi_{1}^{p}(x, \widetilde{\varrho})=\lambda \varphi_{1}^{p}(x, \widetilde{\varrho}), \quad L_{1}^{p} \psi_{1}^{p}(x, \widetilde{\varrho})=\lambda \psi_{1}^{p}(x, \widetilde{\varrho}), \\
\varphi_{1}^{p}(x) \stackrel{x \rightarrow-\infty}{\longrightarrow} 0, \quad \psi_{1}^{p}(x) \stackrel{x \rightarrow+\infty}{\longrightarrow} 0, \quad \widetilde{\varrho}^{2}=\lambda, \quad \operatorname{Re} \widetilde{\varrho}>0, \\
L_{2}^{p} \varphi_{2}^{p}(x, \varrho)=\lambda \varphi_{2}^{p}(x, \varrho), \quad L_{2}^{p} \psi_{2}^{p}(x, \varrho)=\lambda \psi_{2}^{p}(x, \varrho), \\
\varphi_{2}^{p}(x) \stackrel{x \rightarrow-\infty}{\longrightarrow} 0, \quad \psi_{2}^{p}(x) \stackrel{x \rightarrow+\infty}{\longrightarrow} 0, \quad \varrho^{2}=\lambda, \quad \operatorname{Im} \varrho>0 .
\end{gathered}
$$


Рассмотрим также функцию Грина дифференциального оператора $L_{2}$ (см. формулы (7)):

$$
\begin{gathered}
G(x, z, \varrho)=\left(y_{1}\left(\varrho^{2 /(2+\alpha)} z\right) y_{2}\left(\varrho^{2 /(2+\alpha)} x\right)-y_{1}\left(\varrho^{2 /(2+\alpha)} x\right) y_{2}\left(\varrho^{2 /(2+\alpha)} z\right)\right) \varrho^{-2 /(2+\alpha)}, \\
C_{0}=D_{0}=1 .
\end{gathered}
$$

Легко видеть, что $\psi_{2}^{p}(x, \varrho)$ является решением интегрального уравнения

$$
\psi_{2}^{p}(x, \varrho)=\psi_{2}(x, \varrho)+\int_{x}^{+\infty}|z|^{\alpha} G(x, z, \varrho) \varrho^{2}(p(z)-1) \psi_{2}^{p}(z, \varrho) d z
$$

где $\psi_{2}(x, \varrho)$ определяется формулами $(3),(6)$.

Используя формулы $(6),(7),(24)$ и учитьвая специфику операторов $L_{1}$ и $L_{2}$ относительно замены $x \mapsto \varrho^{2 /(2+\alpha)} x$, нетрудно для $x \geqslant 0, \operatorname{Im} \varrho \geqslant 0$ получить оценки

$$
\begin{aligned}
& \left|\psi_{2}(x, \varrho)\right| \leqslant \frac{C}{\left(\left|\varrho^{2 /(2+\alpha)} x\right|^{|\alpha| / 4}+1\right)^{\operatorname{sign} \alpha}} \exp \left\{-\operatorname{Im} \varrho \frac{1}{\alpha / 2+1} x^{\alpha / 2+1}\right\}, \\
& |y(x, \varrho)| \leqslant \frac{C}{\left(\left|\varrho^{2 /(2+\alpha)} x\right|^{|\alpha| / 4}+1\right)^{\operatorname{sign} \alpha}} \exp \left\{+\operatorname{Im} \varrho \frac{1}{\alpha / 2+1} x^{\alpha / 2+1}\right\}, \\
& y_{1}(x, \varrho)=\gamma_{1} \psi_{2}(x, \varrho)+\gamma_{2} y(x, \varrho), \quad y_{2}(x, \varrho)=\gamma_{3} \psi_{2}(x, \varrho)+\gamma_{4} y(x, \varrho),
\end{aligned}
$$

где $\left\{\gamma_{i}\right\}_{i=1}^{4}-$ некоторые не зависящие от $x$ и $\varrho$ константы.

Теперь из формулы (32) при условии (26) стандартными рассуждениями (см., например, [11]-[13]) получаем аналитичность функции $\psi_{2}^{p}$ по $\varrho$ в области $\operatorname{Im} \varrho>0$, непрерывность вплоть до гранищы и оценки $(\operatorname{Im} \varrho \geqslant 0)$ :

$$
\begin{aligned}
\left|\psi_{2}^{p}(x, \varrho)\right| & \leqslant \frac{C}{\left(\left|\varrho^{2 /(2+\alpha)} x\right|^{|\alpha| / 4}+1\right)^{\operatorname{sign} \alpha}} \exp \left\{-\operatorname{Im} \varrho \frac{1}{\alpha / 2+1} x^{\alpha / 2+1}\right\}, \quad x \geqslant 0 \\
\psi_{2}^{p}(x, \varrho) & =(1+o(1)) \frac{1}{\left(\varrho^{2 /(2+\alpha)} x\right)^{\alpha / 4}} \exp \left\{i \varrho \frac{1}{\alpha / 2+1} x^{\alpha / 2+1}\right\} \\
\left(\psi_{2}^{p}\right)^{\prime}(x, \varrho) & =(1+o(1)) \frac{d}{d x}\left(\frac{1}{\left(\varrho^{2 /(2+\alpha)} x\right)^{\alpha / 4}} \exp \left\{i \varrho \frac{1}{\alpha / 2+1} x^{\alpha / 2+1}\right\}\right) \\
x \rightarrow+\infty, \quad \varrho \neq 0, & \\
\psi_{2}^{p}(x, \varrho) & =\psi_{2}(x, \varrho)+o(1), \varrho \rightarrow 0, \quad x \geqslant 0,
\end{aligned}
$$

Аналогичные оценки могут быть выписаны и для $\varphi_{2}^{p}(x, \varrho), \varphi_{1}^{p}(x, \widetilde{\varrho}), \psi_{1}^{p}(x, \widetilde{\varrho})$.

Очевидно, что величина $\lambda=\xi \pm i \varepsilon=\varrho^{2}, \varepsilon>0$, является собственным значением оператора $L$ тогда и только тогда, когда $W^{p}(\varrho, \widetilde{\varrho}= \pm \varrho)=0$. Пусть теперь $\lambda$ - собственное значение. Поскольку $\psi_{2}^{p}(0) \varphi_{1}^{p}(0) \neq 0$ (так как задачи Дирихле и Неймана на полуосях $\mathbb{R}_{ \pm}$для самосопряженных операторов $L_{1}^{p}$ и $L_{2}^{p}$ не имеют невешественных собственных значений), то $\alpha_{+}=\alpha_{-}$, где $\alpha_{+}:=\left(\psi_{2}^{p}\right)^{\prime}(0) / \psi_{2}^{p}(0), \alpha_{-}:=\left(\varphi_{1}^{p}\right)^{\prime}(0) / \varphi_{1}^{p}(0)$. Далее,

$$
\begin{aligned}
& \lambda \int_{0}^{\infty}|x|^{\alpha}\left|\psi_{2}^{p}(x)\right|^{2} d x=-\int_{0}^{\infty}\left(\psi_{2}^{p}\right)^{\prime \prime}(x) \overline{\left(\psi_{2}^{p}\right)}(x) d x=\alpha_{+}\left|\psi_{2}^{p}(0)\right|^{2}+\int_{0}^{\infty}\left|\left(\psi_{2}^{p}\right)^{\prime}(x)\right|^{2} d x \\
& \lambda \int_{-\infty}^{0}|x|^{\alpha}\left|\varphi_{1}^{p}(x)\right|^{2} d x=\int_{-\infty}^{0}\left(\varphi_{1}^{p}\right)^{\prime \prime}(x) \overline{\left(\varphi_{1}^{p}\right)}(x) d x=\alpha_{-}\left|\varphi_{1}^{p}(0)\right|^{2}-\int_{-\infty}^{0}\left|\left(\varphi_{1}^{p}\right)^{\prime}(x)\right|^{2} d x
\end{aligned}
$$


Теперь, выражая $\alpha_{+}$и $\alpha_{-}$и приравнивая вещественные и мнимые части, нетрудно прийти к противоречию, что и доказьвает лемму.

Таким образом, $W^{p}(\varrho) \neq 0$ в области $\{\operatorname{Im} \varrho>0, \operatorname{Re} \varrho>0\}$. Покажем, что аналогичное утверждение справедливо для функций $C_{3}^{p}(\varrho)$ и $B_{3}^{p}(\varrho)$ (определяемых так же, как и $C_{3}, B_{3} ;$ см. $\left.(10),(15)\right)$. В самом деле, как легко видеть, нули функции $C_{3}^{p}(\varrho)$ в рассматриваемой области определяют по формуле $\lambda=\varrho^{2}$ собственные значения оператора $L^{\prime}$ :

$$
L^{\prime}=-\frac{\operatorname{sign} x}{|x|^{\alpha} q(x)} \frac{d^{2}}{d x^{2}}, \quad \text { где } q(x)=p(x), x \geqslant 0, q(x)=p(-x), x<0,
$$

что противоречит лемме 4 . Построения для функции $B_{3}^{p}(\varrho)$ вполне аналогичны.

Заметим далее, что аналогично соотношениям (35) имеет место равенство

$$
\psi_{2}(x, \varrho)=\delta_{1} y_{1}(x, \varrho)+\delta_{2} y_{2}(x, \varrho)
$$

где $\left\{\delta_{i}\right\}_{i=1}^{2}$ - не зависяшие от $x$ и $\varrho$ константы (данное утверждение может служить дополнением к равенствам $(22),(23))$. Более того, $\delta_{1} \cdot \delta_{2} \neq 0$, так как в противном случае либо задача Дирихле, либо задача Неймана на полуоси $\mathbb{R}_{+}$для самосопряженного оператора $L_{2}^{p}$ имела бы невешественные собственные значения. Таким образом, оценки (39), (40) могут быть переписаны в виде

$$
\begin{aligned}
& \psi_{2}^{p}(x, \varrho)=\psi_{2}(x, \varrho)(1+o(1)), \quad|\varrho| \rightarrow 0, \quad \operatorname{Im} \varrho \geqslant 0, \quad x \geqslant 0, \\
& \left(\psi_{2}^{p}\right)^{\prime}(x, \varrho)=\psi_{2}^{\prime}(x, \varrho)(1+o(1)), \quad|\varrho| \rightarrow 0, \quad \operatorname{Im} \varrho \geqslant 0, \quad x \geqslant 0 .
\end{aligned}
$$

ЛЕмма 5. При условии (26) на любом компакте в области $\{\operatorname{Re} \varrho \geqslant 0, \operatorname{Im} \varrho \geqslant 0\}$ выполнены оченки

$$
\left|\frac{W_{3}^{p}(\varrho)}{W^{p}(\varrho)}\right| \leqslant C^{\prime}, \quad\left|\frac{B^{p}(\varrho)}{B_{3}^{p}(\varrho)}\right| \leqslant C^{\prime}, \quad\left|\frac{C^{p}(\varrho)}{C_{3}^{p}(\varrho)}\right| \leqslant C^{\prime} .
$$

ДоказАТЕЛьство. Докажем первое неравенство. С учетом леммы 4 нам достаточно показать вьполнение соответствующей оценки в окрестности $\varrho=0$ и неравенство

$$
W^{p}(\varrho) \neq 0, \quad \varrho \in\left\{\mathbb{R}_{+} \cup i \mathbb{R}_{+}\right\} /\{0\} .
$$

Справедливость оценки в окрестности нуля с очевидностью следует из равенств (3), (22), асимптотик (42), (43) и аналогичньх выражений для $\varphi_{1}^{p}$.

Чтобы получить неравенство (45), достаточно заметить, что $\varphi_{1}^{p}(x, \varrho)=\overline{\left(\varphi_{1}^{p}\right)}(x, \varrho)$ для $\varrho \in \mathbb{R}_{+}$и $\psi_{2}^{p}(x, \varrho)=(-1)^{\alpha /(2(2+\alpha))} \overline{\left(\psi_{2}^{p}\right)}(x, \varrho)$ для $\varrho \in i \mathbb{R}_{+}$. Теперь из $(36)-(38),(41)$ и аналогичных оценок для $\varphi_{1}^{p}$ легко вывести неравенство

$$
\left|\operatorname{Im} W^{p}(\varrho)\right| \geqslant \gamma|\varrho|^{2 /(2+\alpha)}, \quad \gamma>0, \quad \varrho \in\left\{\mathbb{R}_{+} \cup i \mathbb{R}_{+}\right\},
$$

что и доказьвает требуемое утверждение.

Второе и третье неравенства (44) можно доказьвать подобными же рассуждениями или воспользоваться построениями, осуществленными при доказательстве леммы 4. 
Сделаем теперь следующее преобразование исходного уравнения:

$$
\begin{gathered}
\pm \frac{1}{|x|^{\alpha}} y^{\prime \prime}{ }_{x x}(x)=\lambda p(x) y(x) \mapsto \pm \frac{1}{|\xi|^{\alpha}} g^{\prime \prime}{ }_{\xi \xi}(\xi) \mp q(\xi) g(\xi)=\lambda g(\xi) \\
y(x)=\frac{g(\xi)}{\sqrt{u^{\prime}(x)}}, \quad \xi=u(x)
\end{gathered}
$$

где $u(x)$ определяется формулой $(28)$ и

$$
q(\xi)=\left(\frac{3}{4} \frac{\left(u^{\prime \prime}(x)\right)^{2}}{\left(u^{\prime}(x)\right)^{4}}-\frac{1}{2} \frac{u^{\prime \prime \prime}(x)}{\left(u^{\prime}(x)\right)^{3}}\right) \frac{1}{(u(x))^{\alpha}}, \quad x=x(\xi)
$$

(Здесь мы предполагаем, что $\xi \rightarrow \pm \infty$, когда $x \rightarrow \pm \infty$. Заметим, что в условиях теоремы 3 это выполнено автоматически.) Легко видеть, что условие (27) эквивалентно неравенству

$$
\int_{-\infty}^{+\infty} \frac{|\xi|^{\alpha}|q(\xi)|}{\left(|\xi|^{|\alpha| / 2}+1\right)^{\operatorname{sign} \alpha}} d \xi<\infty
$$

Теперь уже нетрудно проверить, что решение интегрального уравнения

$$
g_{1}(\xi, \varrho)=\psi_{2}(\xi, \varrho)-\int_{\xi}^{+\infty}|t|^{\alpha} G(\xi, t, \varrho) q(t) g_{1}(t, \varrho) d t
$$

где $\psi_{2}(\xi, \varrho)$ и $G(\xi, t, \varrho)$ те же, что и раньше, обладает асимптотиками

$$
\begin{aligned}
g_{1}(\xi, \varrho) & =\frac{1}{\left(\varrho^{2 /(2+\alpha)} \xi\right)^{\alpha / 4}} \exp \left\{i \varrho \frac{1}{\alpha / 2+1} \xi^{\alpha / 2+1}\right\}(1+o(1)), \\
x \rightarrow+\infty, \quad \operatorname{Im} \varrho \geqslant 0, \quad \varrho \neq 0, & \\
g_{1}(\xi, \varrho) & =\psi_{2}(\xi, \varrho)(1+o(1)), \quad|\varrho| \rightarrow \infty, \quad \operatorname{Im} \varrho \geqslant 0, \quad \xi \geqslant 0, \\
g_{1}^{\prime}(\xi, \varrho) & =\psi_{2}^{\prime}(\xi, \varrho)(1+o(1)), \quad|\varrho| \rightarrow \infty, \quad \operatorname{Im} \varrho \geqslant 0, \quad \xi \geqslant 0 .
\end{aligned}
$$

Кроме того, очевидно, что функция

$$
\widetilde{\psi}_{2}^{p}(x, \varrho) \equiv \frac{1}{\sqrt{u^{\prime}(x)}} g_{1}(u(x), \varrho)
$$

является решением исходного уравнения $L_{2}^{p} y=\lambda y$. Заметим также, что асимптотики двух решений: $\widetilde{\psi}_{2}^{p}(x, \varrho)$ и $\psi_{2}^{p}(x, \varrho), x \rightarrow+\infty($ см. $(28),(37),(48),(51))$, являются экспоненциально убывающими, когда $\operatorname{Im} \varrho>0$. Таким образом, учитьвая единственность решения с экспоненциально убьвающей асимптотикой, мы приходим к равенству

$$
\psi_{2}^{p}(x, \varrho)=C(\varrho) \widetilde{\psi}_{2}^{p}(x, \varrho), \quad \operatorname{Im} \varrho>0
$$

где $C(\varrho)$ - некоторая локально ограниченная, не имеющая нулей функция от $\varrho$ в области $\operatorname{Im} \varrho>0$.

ЛЕмма 6. В условиях теоремы 3 неравенства (44) справедливы во всей облас$m u\{\operatorname{Im} \varrho \geqslant 0, \operatorname{Re} \varrho \geqslant 0\}$. 
ДокАЗАТЕЛЬСтво. С учетом леммы 5 , а также замечания относительно второго и третьего неравенств (44) для доказательства леммы 6 нам достаточно получить оценку

$$
\left|\frac{W_{3}^{p}(\varrho)}{W^{p}(\varrho)}\right| \leqslant C^{\prime}, \quad|\varrho| \rightarrow \infty, \quad \operatorname{Im} \varrho \geqslant 0, \quad \operatorname{Re} \varrho \geqslant 0 .
$$

Последнее легко следует из предыдущих построений. Действительно, равенство (52) совместно с (49)-(51) дает асимптотики

$$
\begin{aligned}
& \psi_{2}^{p}(x, \varrho)=C(\varrho) \frac{1}{\left(u^{\prime}(x)\right)^{1 / 2}} \psi_{2}(u(x), \varrho)(1+o(1)), \quad|\varrho| \rightarrow \infty, \operatorname{Im} \varrho>0, \quad x \geqslant 0 \\
&\left(\psi_{2}^{p}\right)^{\prime}(x, \varrho)=C(\varrho)\left(\left(u^{\prime}(x)\right)^{1 / 2} \frac{d}{d \xi} \psi_{2}(\xi, \varrho)-\frac{1}{2} \frac{u^{\prime \prime}(x)}{\left(u^{\prime}(x)\right)^{3 / 2}} \psi_{2}(u(x), \varrho)\right)(1+o(1)) \\
&|\varrho| \rightarrow \infty, \quad \operatorname{Im} \varrho>0, \quad x \geqslant 0 .
\end{aligned}
$$

Полагая в формулах $(54),(55) x=+0$ и учитьвая, что $\xi(+0) \equiv u(+0)=0($ см. $(28))$, получаем

$$
\begin{gathered}
\psi_{2}^{p}(0, \varrho)=C(\varrho) \frac{1}{\left(u^{\prime}(+0)\right)^{1 / 2}} \psi_{2}(0, \varrho)(1+o(1)), \quad|\varrho| \rightarrow \infty, \quad \operatorname{Im} \varrho>0 \\
\left(\psi_{2}^{p}\right)^{\prime}(0, \varrho)=C(\varrho)\left(\left(u^{\prime}(+0)\right)^{1 / 2} \psi_{2}^{\prime}(0, \varrho)-\frac{1}{2} \frac{u^{\prime \prime}(+0)}{\left(u^{\prime}(+0)\right)^{3 / 2}} \psi_{2}(0, \varrho)\right)(1+o(1)), \\
|\varrho| \rightarrow \infty, \quad \operatorname{Im} \varrho>0 .
\end{gathered}
$$

Аналогично можно получить

$$
\begin{gathered}
\varphi_{1}^{p}(0, \widetilde{\varrho})=C^{\prime}(\widetilde{\varrho}) \frac{1}{\left(u^{\prime}(-0)\right)^{1 / 2}} \varphi_{1}(0, \widetilde{\varrho})(1+o(1)), \quad \mid \widetilde{\varrho} \rightarrow \infty, \operatorname{Re} \widetilde{\varrho}>0, \\
\left(\varphi_{1}^{p}\right)^{\prime}(0, \widetilde{\varrho})=C^{\prime}(\widetilde{\varrho})\left(\left(u^{\prime}(-0)\right)^{1 / 2} \varphi_{1}^{\prime}(0, \widetilde{\varrho})-\frac{1}{2} \frac{u^{\prime \prime}(-0)}{\left(u^{\prime}(-0)\right)^{3 / 2}} \varphi_{1}(0, \widetilde{\varrho})\right)(1+o(1)), \\
|\widetilde{\varrho}| \rightarrow \infty, \operatorname{Re} \widetilde{\varrho}>0,
\end{gathered}
$$

где $C^{\prime}(\widetilde{\varrho})$ - локально ограниченная, не имеющая нулей функция от $\widetilde{\varrho}$ в области $\operatorname{Re} \widetilde{\varrho}>0$.

Заметим, что $u^{\prime}(+0)=u^{\prime}(-0) \neq 0$, если $p(+0)=p(-0) \neq 0$, и, следовательно (см. (6), (41)),

$$
\begin{array}{ll}
W^{p}(\varrho)=C(\varrho) C^{\prime}(\varrho) W(\varrho)(1+o(1)), & |\varrho| \rightarrow \infty, \quad \operatorname{Im} \varrho>0, \quad \operatorname{Re} \varrho>0 \\
W_{3}^{p}(\varrho)=C(\varrho) C^{\prime}(\varrho) W_{3}(\varrho)(1+o(1)), & |\varrho| \rightarrow \infty, \quad \operatorname{Im} \varrho>0, \quad \operatorname{Re} \varrho>0 .
\end{array}
$$

Теперь утверждение леммы 6 немедленно следует из формул $(3),(22),(60),(61)$ и непрерьвности функции $W_{3}^{p}(\varrho) / W^{p}(\varrho)$ в области $\{\operatorname{Im} \varrho \geqslant 0, \operatorname{Re} \varrho \geqslant 0, \varrho \neq 0\}$ (см. формулу (45) и рассуждения, предшествующие лемме 5).

Нам осталось лишь заметить, что рассуждения, аналогичные проведенным при доказательстве теоремы 1 , показьвают, что для подобия оператора $L$ самосопряженному оператору достаточно вьполнения оценок (44) в области $\{\operatorname{Im} \varrho \geqslant 0, \operatorname{Re} \varrho \geqslant 0\}$. Таким образом, применение леммы 6 и завершает доказательство теоремы 3.

Заметим, что условия теоремы 3 могут быть ослаблены. Именно, имеет место следующее утверждение. 
Теорема 7. Пусть $\alpha>-1,0<c \leqslant p(x) \leqslant C<\infty ; p(x), p^{\prime}(x)-$ локально абсолютно непрерывны на $\mathbb{R}_{ \pm}$и выполнены условия

$$
\int_{0}^{+\infty} \frac{|x|^{\alpha}\left|p(x)-c_{+}\right|}{\left(|x|^{|\alpha| / 2}+1\right)^{\operatorname{sign} \alpha}} d x<\infty, \quad \int_{-\infty}^{0} \frac{|x|^{\alpha}\left|p(x)-c_{-}\right|}{\left(|x|^{|\alpha| / 2}+1\right)^{\operatorname{sign} \alpha}} d x<\infty
$$

əде $c_{-}>0, c_{+}>0, u$

$$
\int_{-\infty}^{+\infty} \frac{\left|3\left(u^{\prime \prime}(x)\right)^{2} / 2 u^{\prime}(x)-u^{\prime \prime \prime}(x)\right|}{\left(u^{\prime}(x)\right)^{2}\left(|u(x)|^{|\alpha| / 2}+1\right)^{\operatorname{sign} \alpha}} d x<\infty
$$

где и(x) определяется формулой (28). Тогда оператор L подобен самосопряженному оператору.

Доказательство теоремы 7 вполне аналогично предыдущим построениям и требует лишь несколько более тщательного анализа.

В заключение авторы выражают свою благодарность С.Н. Набоко за внимание к работе и В.В. Капустину за полезные обсуждения.

\section{СПИСОК ЦИТИРОВАННОЙ ЛИТЕРАТУРЫ}

[1] Fleige A., Najman B. Nonsingularity of critical points of some differential and difference operators // Operators Theories: Advances and Appl. 1998. V. 106. P. 147-155.

[2] Langer H. Spectral function of definitizable operators in Krein spaces // Functional Analysis. Proceedings (Dubrovnik 1981). Lecture Notes in Math. V. 948. Berlin: Springer Verlag, 1982. P. 1-46.

[3] Фаддеев М.М., Штеренберг Р. Г. О подобии некоторых сингулярных дифференциальных операторов самосопряженным // Исследования по линейным операторам и теории функций. 28 (Записки научных семинаров ПОМИ. Т. 270). Сб. работ под ред. С. В. Кислякова. СПб.: ПОМИ РАН, 2000. С. 336-349.

[4] Карабаш И. М. О J-самосопряженных дифференциальных операторах, подобных самосопряженньм // Матем. заметки. 2000. Т. 68. №6. С. 943-944.

[5] Маламуд М. М. О подобии треугольного оператора диагональному // Исследования по линейным операторам и теории функций. 28 (Записки научных семинаров ПОМИ. Т. 270). Сб. работ под ред. С. В. Кислякова. СПб.: ПОМИ РАН, 2000. С. 201-241.

[6] Сахнович Л.А. Неунитарные операторы с абсолютно непрерывным спектром // Изв. АН CССР. Сер. матем. 1969. Т. 33. №1. С. 52-64.

[7] Секефальви-Надь Б., Фолш Ч. Гармонический анализ операторов в гильбертовом пространстве. М.: Мир, 1970.

[8] Van Casteren J. Operators similar to unitary or self-adjoint ones // Pacific J. Math. 1983. V. 104. P. 241-255.

[9] Набоко С. Н. Об условиях подобия унитарным и самосопряженным операторам // Функцион. анализ и его прилож. 1984. Т. 18. № 1. С. 16-27.

[10] Агранович 3. С., Марченко В. А. Обратная задача теории рассеяния. Харьков, 1960.

[11] Блащак В.В. О разложении по главным функциям несамосопряженного дифференциального оператора второго порядка на всей оси // Докл. АН УРСР. 1965. № 4. С. 416-419.

[12] Блащак В.В. О дифференциальном операторе второго порядка на всей оси со спектральными особенностями // Докл. АН УРСР. 1966. № 1. С. 38-41.

[13] Лянце В. Э. О несамосопряженном дифференциальном операторе второго порядка на полуоси // Докл. АН СССР. 1964. Т. 154. № 5. С. 1030-1033. 